\title{
TRACERx: Tracking tumor evolution to impact the course of lung cancer
}

\footnotetext{
From the ${ }^{\mathrm{a}}$ Division of Cancer Medicine, Department of Thoracic/Head and Neck Medical Oncology, ${ }^{\mathrm{b}}$ Division of Cancer Medicine, Department of Genomic Medicine, and ${ }^{\mathrm{c}}$ Division of Surgery, Department of Thoracic and Cardiovascular Surgery, The University of Texas MD Anderson Cancer Center, Houston, Tex.

Funded by the MD Anderson Lung Cancer Moon Shots Program, MD Anderson Physician Scientist Program, and

The Cancer Prevention \& Research Institute of Texas Multi-Investigator Research Award grant (RP160668). Drs Negrao and Quek contributed equally to this article.

Received for publication Sept 5, 2017; revisions received Sept 27, 2017; accepted for publication Oct 11, 2017; available ahead of print Jan 11, 2018.

Address for reprints: Boris Sepesi, MD, T. Boone Pickens Academic Towe (FCT19.5084), 1515 Holcombe Blvd, Unit 1489, Houston, TX 77030 (E-mail: BSepesi@mdanderson.org).

J Thorac Cardiovasc Surg 2018;155:1199-202

$0022-5223 / \$ 36.00$

Copyright (C 2017 by The American Association for Thoracic Surgery

https://doi.org/10.1016/j.jtcvs.2017.10.134
}

Marcelo Vailati Negrao, MD, ${ }^{a}$ Kelly Quek, $\mathrm{PhD},{ }^{\mathrm{b}}$ Jianjun Zhang, MD, PhD, ${ }^{\mathrm{a}, \mathrm{b}}$ and Boris Sepesi, $\mathrm{MD}^{\mathrm{c}}$
Feature Editor's Note- "When the views entertained in this volume on the origin of species, or when analogous views are generally admitted, we can dimly forsee that there will be a considerable revolution in natural history."

\section{-Charles Darwin}

Recent landmark studies on intratumoral heterogeneity require us to revise our current conceptual framework for understanding the evolution of cancer. Whole-exome sequencing on multiple regions of single human tumors has demonstrated striking intraclonal diversity in cancer: whereas some somatic mutations are clonal in nature (present in all tumor cells), many are subclonal (present in only some tumor cells). Critical to our understanding of cancer as a complex adaptive and evolving biologic ecosystem, these discoveries have demonstrated that cancer evolves not on a linear trajectory but through parallel evolution of subclones. Thus, intratumoral heterogeneity underlies, and fuels, tumor evolution. This concept has vital clinical implications for patients suffering from cancer, particularly in areas of targeted therapy and drug resistance. For example, an "actionable" driver mutation may be a suboptimal target for therapy if confined to a subclonal branch of the tumor's evolutionary tree. In the accompanying Feature Editorial, the authors discuss the major findings of the TRACERx (Tracking Non-Small Cell Lung Cancer Evolution through Therapy) study recently published in The New England Journal of Medicine, in which the nature of intratumoral heterogeneity is defined for 100 patients with non-small cell lung cancer treated surgically within a multicenter cohort. The findings, reported herein, are striking, and will drive innovation in the prevention, diagnosis, and treatment of lung, and all, human cancers. We can expect to see our own rapid evolution of this field, from which we ultimately can hope adaptiveness.

\section{Bryan Burt, $M D$}

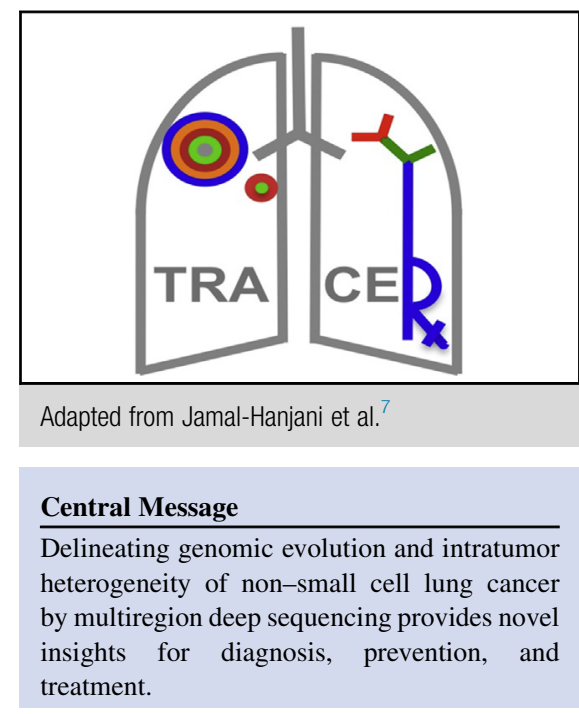

See Editorial Commentary page 1203.

to provide therapeutic interventions that reduce tumor

Non-small cell lung cancer (NSCLC) continues to be a prevalent and deadly cancer, and the variability of recurrences argues for other tumor, patient, or treatmentrelated factors beyond current staging, which influence prognosis. One significant aspect of lung cancer biology that has received increased attention relates to the heterogeneity and evolution of the tumor itself.

Tumor heterogeneity results from tumor evolution and in the meanwhile serves as a substrate for tumor evolution and natural selection and is influenced by the host system (such as antitumor immune surveillance) as well as anticancer therapies. Delineating cancer evolutionary history may provide pivotal insight to our understanding of cancer development, progression, and therapeutic resistance. Pioneering studies by multiregion profiling ${ }^{1-4}$ and by comparing paired primary and relapsed tumors ${ }^{5,6}$ have shed light on cancer genomic evolution and suggested the potential impact of genomic heterogeneity on cancer biology and patient outcome. However, these studies have been limited to small retrospective cohorts.

The Tracking Non-Small Cell Lung Cancer Evolution through Therapy (TRACERx) study ${ }^{7}$ is an ambitious study 
targeting the evolution of NSCLC. It is a multicenter cohort study aiming to prospectively evaluate tumor evolution of 842 patients with NSCLC treated initially with surgical therapy. The objective is to examine the tumor heterogeneity at the time of surgical resection, at relapse, and after sequential lines of other treatments through high-depth, multiregion whole-exome sequencing of surgically resected specimen and sequentially collected tumor biopsy samples at the time of relapse. Results are also correlated with plasma circulating tumor DNA (ctDNA), which is analyzed from the blood samples collected throughout the patient's clinical course.

The results from the first 100 patients (stage IA-IIIB) enrolled in TRACERx study were recently published in The New England Journal of Medicine. ${ }^{7}$ This report was directed at investigating the intratumoral heterogeneity (ITH) architecture of surgically excised tumors and evaluating whether ITH is associated with clinical outcome. In this elegant analysis, 327 tumor regions from 100 tumors were subjected to high-depth whole-exome sequencing (median sequencing depth of $426 \times$ ) and sophisticated bioinformatics analyses. The authors demonstrated extensive genomic ITH at the nucleotide and chromosomal level; the median of $30 \%$ of somatic mutations and the median of $48 \%$ of copy number alterations (CNAs) were subclonal. Lung squamous cell carcinoma demonstrated greater clonal mutational burden than lung adenocarcinoma, probably reflecting heavier smoking history in patients with lung squamous cell carcinoma; however, subclonal mutation burden or ratio did not seem to differ between the histologies.

As expected, early clonal mutations were associated with smoking (COSMIC signature 4) in the majority of tumors. In contrast, subclonal mutations were significantly enriched for genomic signatures related to spontaneous deamination of methylated cytosines (COSMIC signature 1A) and APOBEC (apolipoprotein B mRNA editing enzyme, catalytic polypeptide-like) (COSMIC signatures 2 and 13), ${ }^{8}$ suggesting continuous genomic evolution with different mutational mechanisms in play, which occurred at different times during cancer development and progression.

The authors also tested their central hypothesis whether ITH is associated with clinical outcome. Their preliminary analysis demonstrated that a high proportion $(\geq 48 \%$, the cohort median) of subclonal CNAs was associated with worse disease-free survival (DFS) as compared with a low proportion of subclonal CNAs. This finding remained significant after adjustment for age, smoking, histology, adjuvant therapy, and tumor stage (hazard ratio, 3.70; $95 \%$ confidence interval, 1.29-10.65; $P=.01$ ). However, no significant association with DFS was observed between the groups when stratified by percentage of subclonal mutations.

Although the published work is only the preliminary analysis of the first 100 of proposed 842 patients, the rich information and novel findings are quite provocative for the future translational and clinical research. Herein, we outline some of the concepts.

First, this work provided another piece of evidence that genomic heterogeneity is associated with clinical outcome, even in early stages of NSCLC. It is somewhat surprising that more commonly regarded ITH in point mutations was not found to be associated with survival in this patient cohort; this finding is in contrast with previous reports in lung cancer ${ }^{1,2}$ and other malignancies. ${ }^{9}$ One possible explanation is that the imbalance in many major prognostic factors, such as stage, age, smoking, and adjuvant therapy, in this relative small patient cohort may have masked an actual association between mutational ITH and survival. In addition, there have been only 20 relapses at the time of data analyses, and the postsurgical follow-up of this study has been rather short. The median followup duration for the cohort has been approximately 18 months and less than 2 years in 80 patients. Therefore, it is premature to conclude that point-mutation ITH is not associated with survival in locoregional NSCLC. Hopefully, this question will be more definitively answered when additional patients from TRACERx or similar studies are analyzed after prolonged follow-up. Nevertheless, the association between the high level of CNA ITH and worse DFS, which remained significant on a multivariate analysis after adjusting for known prognostic factors, suggests that chromosomal instability may have greater impact on outcome than somatic mutations. Chromosomal alterations involving the gain or loss of chromosomal segments or even whole chromosomes could affect hundreds or thousands of genes and may thus disrupt multiple key molecular processes.

Second, by the use of multiregion sequencing, subclonal driver mutations were identified in $75 \%$ of patients in this study. This introduces a challenge to our current personalized oncology approach, which is based on sequencing driver genes from single biopsies acquired either by bronchoscopy or guided transcutaneous biopsy. Multiregion sequencing is not practical for patients with metastatic diseases or unresectable tumors. However, ctDNA is not spatially limited to certain tumor regions and may have the advantage in detecting subclonal mutations compared with single biopsies. Indeed, in an accompanying article published in Nature,$^{10}$ the TRACERx team has investigated the role of ctDNA in molecular diagnosis and disease monitoring and was able to identify clonal and 
subclonal mutations present in matched tumor samples. With the rapid progress being made in liquid biopsy sequencing technologies, sequencing ctDNA could become a practical alternative approach to multiregion tumor sequencing.

Third, in this study all tumors were primarily resected. How neoadjuvant therapies such as chemotherapy, targeted therapy, radiation, or immune therapy would impact ITH architectures of NSCLC remains unknown. One can hypothesize that residual tumor cells that survive neoadjuvant therapies could be the subclones resistant to neoadjuvant therapies. Therefore, investigating the postneoadjuvant therapy residual tumors may provide valuable information on mechanisms of drug resistance. For example, because targetable driver mutations such as EGFR, MET, and BRAF are all clonal, the residual cancer cells in the resected tumors and lymph nodes after appropriate targeted treatment would provide valuable material to investigate the mechanisms underlying resistance to these agents. Therefore, well-designed window-of-opportunity neoadjuvant clinical trials would be valuable for studying drug resistance.

Fourth, in addition to serving as a potential prognostic biomarker, ITH itself could become a potential therapeutic target. Given the important role of genomic instability in tumor evolution, modulating genomic stability such as targeting APOBEC family, a common cause of subclonal diversification of NSCLC, or inhibiting DNA repair pathways could become a novel therapeutic strategy. This strategy has been recently tested. For example, poly (ADP-ribose) polymerase inhibitors have proven to be active in homologous recombination-deficient tumors. ${ }^{11}$

Fifth, the majority of studies on ITH, including the current study, have mainly focused on the genomic ITH. However, ITH can be present at different molecular levels (genetic, epigenetic, gene, and protein expression, etc) of cancer cells and also in tumor microenvironment constituting of epithelial cells, blood and lymphatic vessels, cytokines, or infiltrating immune cells. ITH of any of the aforementioned components may impact tumor evolution and patient outcome. Our pilot study has demonstrated that a greater level of DNA methylation ITH was associated with larger tumor size, advanced patient age, and increased risk of postsurgical recurrence in NSCLC. ${ }^{12}$ Most recently, we reported substantial intratumor differences in T-cell density and clonality with the majority of T-cell clones restricted to individual tumor regions from localized lung cancers and that a greater degree of TCR ITH was associated with an increased risk of postsurgical recurrence and shorter DFS. ${ }^{13}$ Tumor evolution is a complex process, during which cancer cells accumulate molecular alterations that change their phenotypic features by interacting with the tumor microenvironment. To systematically understand tumor evolution, future studies are required to depict the overall molecular (genetic, epigenetic, gene, and protein expression) ITH of cancer cells as well as the tumor microenvironment, particularly the immune contexture. Again, well-designed trials/projects that include surgical locoregional disease control of lung cancer will be invaluable in sorting out this process.

Last but not least, the TRACERx data show a possible molecular poor prognostic marker for lung cancer, in this case high-level CNA ITH, which could be prospectively validated in the future. In the past, clinical, pathologic, and radiologic prognostic markers for NSCLC have not translated into strategies for treatment intensification in high-risk patients, and we continue to use a "one-size fits all" approach. One of the reasons for this was probably the limited number of effective agents, but the therapeutic arsenal for cancer treatment has drastically changed over the last years, especially for NSCLC, where targeted and immune therapies are now approved. Therefore, validated molecular markers and mutational signatures from studies such as TRACERx could play a major role in patient selection in future trials aiming to intensify neoadjuvant and adjuvant treatment for localized or locally advanced disease.

The TRACERx study is an important step to advance our understanding of NSCLC tumor evolution and its impact on cancer biology and patient outcome. We are looking forward to the updated analyses of the study and more such efforts to dissect the evolutionary history of lung cancer and other malignancies, leading to novel diagnostic, preventive, and therapeutic strategies and eventually cure.

\section{Conflict of Interest Statement}

Authors have nothing to disclose with regard to commercial support.

\section{References}

1. Zhang J, Fujimoto J, Zhang J, Wedge DC, Song X, Zhang J, et al. Intratumor heterogeneity in localized lung adenocarcinomas delineated by multiregion sequencing. Science. 2014;346:256-9.

2. McGranahan N, Furness AJS, Rosenthal R, Ramskov S, Lyngaa R, Saini SK, et al. Clonal neoantigens elicit $\mathrm{T}$ cell immunoreactivity and sensitivity to immune checkpoint blockade. Science. 2016;351:1463-9.

3. Gerlinger M, Rowan AJ, Horswell S, Math M, Larkin J, Endesfelder D, et al. Intratumor heterogeneity and branched evolution revealed by multiregion sequencing. N Engl J Med. 2012;366:883-92.

4. Liu Y, Zhang J, Li L, Yin G, Zhang J, Zheng S, et al. Genomic heterogeneity of multiple synchronous lung cancer. Nat Commun. 2016;7:13200.

5. Paik PK, Shen R, Won H, Rekhtman N, Wang L, Sima CS, et al. Next-generation sequencing of stage IV squamous cell lung cancers reveals an association of PI3K aberrations and evidence of clonal heterogeneity in patients with brain metastases. Cancer Discov. 2015;5:610-21. 
6. Brastianos PK, Carter SL, Santagata S, Cahill DP, Taylor-Weiner A, Jones RT, et al. Genomic characterization of brain metastases reveals branched evolution and potential therapeutic targets. Cancer Discov. 2015;5:1164-77.

7. Jamal-Hanjani M, Wilson GA, McGranahan N, Birkbak NJ, Watkins TBK, Veeriah S, et al. Tracking the evolution of non-small-cell lung cancer. $N$ Engl J Med. 2017:376:2109-21.

8. Alexandrov LB, Nik-Zainal S, Wedge DC, Aparicio SA, Behjati S, Biankin AV, et al. Signatures of mutational processes in human cancer. Nature. 2013;500: 415-21.

9. Landau DA, Carter SL, Stojanov P, McKenna A, Stevenson K, Lawrence MS, et al. Evolution and impact of subclonal mutations in chronic lymphocytic leukemia. Cell. 2013;152:714-26.
10. Abbosh C, Birkbak NJ, Wilson GA, Jamal-Hanjani M, Constantin T, Salari R, et al. Phylogenetic ctDNA analysis depicts early-stage lung cancer evolution. Nature. 2017;545:446-51.

11. Mirza MR, Monk BJ, Herrstedt J, Oza AM, Mahner S, Redondo A, et al Niraparib maintenance therapy in platinum-sensitive, recurrent ovarian cancer N Engl J Med. 2016;375:2154-64.

12. Quek K, Li J, Estecio M, Zhang J, Fujimoto J, Roarty E, et al. DNA methylation intratumor heterogeneity in localized lung adenocarcinomas. Oncotarget. 2017;8:21994-2002.

13. Reuben A, Gittelman RM, Gao J, Zhang J, Yusko EC, Wu CJ, et al. TCR repertoire intratumor heterogeneity in localized lung adenocarcinomas: an association with predicted neoantigen heterogeneity and postsurgical recurrence. Cancer Discov. 2017;7:1088-97 